\title{
An Emerging Paradigm for the Integration of Mechanics and Imaging for Material Characterization
}

\author{
Dr. Adil Al-Mayah \\ University of Waterloo, Canada
}

Materials research plays a vital role in the advancement of a broad range of seemingly disparate applications, spanning structures, pavement, automotive and emerging biomedical technologies. For example, Canada's aging infrastructure is deteriorating at a rate that requires immediate repair or renewal, placing a huge strain on our economy and highlighting a critical need for new methods to evaluate and delay structural materials failure.

A new imaging-mechanics-integration technique has been applied to shift material characterization from the conventional 'overall' evaluation to a 'components-based' characterization. Using this approach, high resolution 3D images of a sample are acquired using a high resolution and energy computed tomography (CT) imaging system. Essential morphological and spatial distribution data of internal components, cracks, voids, corrosion products are collected. A sample-specific image-based finite element models can be created to obtain accurate image-based stress distribution and deformation maps to identify failure initiation and crack propagation inside the sample. This approach has a proven potential to make significant advancements in areas of composite material repair applications, asphalt and concrete material investigation, and innovative biomechanical characterization of soft tissues. 